\title{
Testing the translational-symmetry hypothesis of abstract-concept learning in pigeons
}

\author{
JEFFREY S. KATZ \\ Auburn University, Auburn, Alabama \\ BRADley R. STURZ \\ Armstrong Atlantic State University, Savannah, Georgia \\ AND \\ Anthony A. Wright \\ University of Texas Health Science Center, Houston, Texas
}

\begin{abstract}
The translational-symmetry hypothesis of abstract-concept learning was tested in a same/different (S/D) task with pairs of pictures. The translational-symmetry hypothesis proposes that subjects discriminate same trials by the simultaneous repetition of features in the two pictures (and different trials by the lack of feature repetition). Pigeons that had learned a simultaneous S/D task were tested with delays between the two pictures to remove emergent perceptual cues. In Experiment 1, we tested delays of 0 and $1 \mathrm{sec}$. The results did not show the accuracy decrease expected according to the translational-symmetry hypothesis. In Experiment 2, we expanded the delays to 2 and $6 \mathrm{sec}$. Even at the longest delay, there was no evidence of the precipitous performance decline or default strategy that would be predicted by translational symmetry. The results provide evidence against translational symmetry (or other perceptually emergent features) that might control these pigeons' performance in our two-item $\mathrm{S} / \mathrm{D}$ task.
\end{abstract}

The ability to form abstract concepts is fundamental to human thinking (e.g., James, 1890/1950; Penn, Holyoak, \& Povinelli, 2008; Piaget \& Inhelder, 1969). At the center of research on abstract-concept learning is the same/ different (S/D) concept, dating back to William James, who claimed that the "sense of sameness is the very keel and backbone of our thinking" (1890/1950, p. 459). In addition to humans, there has been an animal-cognition focus on which (nonhuman) animal species can and cannot learn S/D abstract concepts, dating back to Premack (1978). Newer and better testing techniques have shown $\mathrm{S} / \mathrm{D}$ abstract-concept learning in one animal, pigeons, thought incapable of S/D abstract-concept learning (e.g., Blaisdell \& Cook, 2005; Cook, Katz, \& Cavoto, 1997; Cook, Kelly, \& Katz, 2003; Cook \& Wasserman, 2006; Katz \& Wright, 2006; Katz, Wright, \& Bodily, 2007; Santiago \& Wright, 1984; Young \& Wasserman, 1997).

New breakthroughs in research, particularly concerning so-called higher order cognitive abilities, are often received with skepticism. Some of the pigeon S/D abstract-concept learning experiments were conducted with arrays (e.g., 16) of icons or letters (Cook, Cavoto, \& Cavoto, 1995; Wasserman, Hugart, \& Kirkpatrick-Steger, 1995). Regarding those findings, one critic, Mackintosh (2000), remarked that "an obvious difference between a $4 \times 4$ array of As and a $4 \times 4$ array consisting of 16 letters $A$ to $P$ each occurring once, is that the former has a regular texture and the latter does not" (p. 132). The phrase "regular texture" refers to the perception of the repetition of stimulus features (and is related to translational symmetry; Mach, 1890/1980; Tyler, 2002). One can lessen the impression of regularity/ translational symmetry in texture arrays by jittering the items (e.g., Humphreys, Quinlan, \& Riddoch, 1989), but some degree of translational symmetry is always present when stimuli are presented simultaneously in the visual field, even if the stimuli are spaced apart. Moreover, the inability of those pigeons to perform accurately when the arrays were reduced to two items would seem to support this criticism, because this cue of translational symmetry would be considerably diminished by using only two items, as is shown in the examples of Figure 1.

In our S/D experiments, we used only two items; two of the pairs that we have used are shown as examples in the top panel of Figure 1 (e.g., Elmore, Wright, Rivera, \& Katz, 2009; Katz \& Wright, 2006; Nakamura, Wright, Katz, Bodily, \& Sturz, 2009; Wright \& Katz, 2006, 2007, 2009). In our S/D experiments, if the two items were same, the correct response was to peck the lower item. If the two items were different, the correct response was to peck a white rectangle (to the right of the lower item). Typically, we trained with an initial set of eight items that was progressively expanded. After each training set's doubling, 




\section{Enhanced Translational Symmetry}

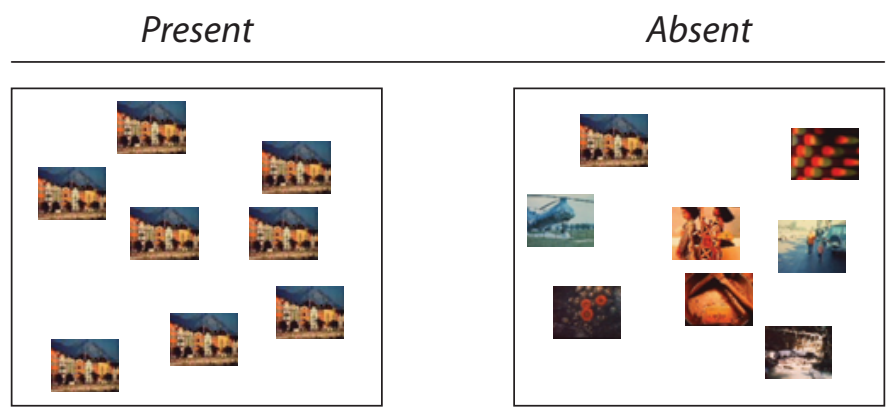

Figure 1. The stimulus displays (top panel) used to train the pigeons. The actual stimulus displays did not have labels, were in full color, and had black backgrounds, and the gray area to the right was white. The impression of translation symmetry occurs in same displays because of the repetition of stimulus features. Translational symmetry becomes stronger when more repetition occurs (bottom panel), even when the stimuli are not vertically or horizontally aligned.

the pigeons were trained to criterion and then tested for novel-stimulus transfer. Novel-stimulus transfer increased progressively along with the size of the training set. Novel-stimulus transfer, the measure of abstract-concept learning, was equivalent to training performance when the training set reached 256 pictures (Katz \& Wright, 2006).

Although translational symmetry might be minimized in our abstract-concept learning experiments with two items, it would not be completely eliminated, as is shown by the examples in Figure 1. Therefore, the purpose of the experiments in this article was to test whether the cue of translational symmetry might in any material way contribute to the accurate performance or novel-stimulus transfer in our two-item pigeon $\mathrm{S} / \mathrm{D}$ experiments.

\section{EXPERIMENT 1}

We tested for the possibility that the cue of translational symmetry might be functional in our pigeon S/D experiments by transferring our subjects to a delayed S/D task, where the top item was removed before presenting the second item. When stimuli are presented sequentially, any emergent features such as translational symmetry should rapidly become imperceptible and therefore would not contribute to accurate performance (cf. Cook \& Wasserman, 2007; Young \& Wasserman, 2001). Indeed, if translational symmetry was critical in our experiments, performance should fall precipitously (possibly to chance, 50\%) at the point at which the emergent regularity feature failed to appear. Another prediction of translational-symmetry hypothesis is that when this emergent feature no longer appeared (by introducing a delay), there should be an im- mediate increase in different responding. The rationale for this prediction follows from the emergent feature of translational symmetry (according to the hypothesis) being the cue for same responses, and its absence (by default) being the cue for different responses. A related prediction is that the pigeons' accurate novel-stimulus transfer performance under the simultaneous presentation of novel test stimuli should disappear under delay testing and should show a dramatic rise in different responding to novel test pairs.

\section{Method}

\section{Subjects}

Four White Carneaux pigeons (Columba livia), 5-6 years old, with previous experience in the S/D task (see Katz \& Wright, 2006), from the Palmetto Pigeon Plant in Sumter, South Carolina, served as subjects. The pigeons were maintained at $80 \%-85 \%$ of their free-feeding weights and were individually housed with a 12:12-h light:dark cycle. Testing was conducted 5-7 days a week during the light phase of the cycle.

\section{Apparatus}

Chamber. The pigeons were tested in a custom wooden chamber (35.9 cm wide $\times 45.7 \mathrm{~cm}$ deep $\times 51.4 \mathrm{~cm}$ high). A custom-built wooden grain hopper allowed a video monitor (Eizo T550, Ishikawa, Japan; $800 \times 600$ pixel resolution) and infrared touchscreen (17-in. Unitouch, Carroll Touch, Round Rock, TX) to be better positioned (i.e., lower) on the stimulus panel. Experimental events were controlled and recorded with custom software written in Visual Basic on a Pentium personal computer. A video card (ATI 3D Rage Pro AGP 2X, Ontario, Canada) controlled the monitor. A computercontrolled relay interface (Model PI0-12, Metrabyte, Taunton, MA) operated the food hopper and houselight.

Stimuli. The stimulus displays consisted of two digitized travelslide color pictures (each $5.7 \times 3.8 \mathrm{~cm}$ ) and a white rectangle $(2.6 \times 2.3 \mathrm{~cm})$ on a black background. The pictures were vertically 
aligned with a $1.28-\mathrm{cm}$ gap between them. The top picture was centered $20.63 \mathrm{~cm}$ from the left edge and $18.75 \mathrm{~cm}$ from the top of the cutout in the front panel. The bottom of the white rectangle was horizontally aligned with the bottom of the lower picture and to the right with a 1.4-cm gap between them. The 1,024 training stimuli were those used by Katz and Wright (2006), and color reproductions of these stimuli can be seen in Wright and Katz (2006).

\section{Procedure}

Sequential S/D task training: 0-sec delay. The pigeons were previously trained in the simultaneous S/D task and showed full concept learning as demonstrated by Katz and Wright (2006). We then trained these pigeons in a sequential S/D task. Specifically, training began with presentation of a picture in the upper position. A response requirement of 20 pecks to this picture resulted in the removal of the upper item followed by a 0 -sec delay. After the delay, the lower picture and the white rectangle were presented. If the two pictures were same (as they were on half of the trials), a peck to the lower picture was correct. If the two pictures were different (as they were on the other half of the trials), a peck to the white rectangle was correct. Correct responses were reinforced with a $0.5-\mathrm{sec}, 600.6-\mathrm{Hz}$ tone and food reinforcement (3-5 sec access to mixed grain). Food reinforcement or an incorrect response was immediately followed by a 15 -sec intertrial interval.

Sessions consisted of 100 (50 same, 50 different) trials. The sequence of same and different trials was randomly constructed and varied from session to session. The items used to construct the displays were selected with replacement from the 1,024-item set. The subjects were trained until performance was $\geq 80 \%$ in a single session. The subjects then immediately started 1 -sec delay training.

Sequential S/D task training: 1-sec delay. Training was identical to the 0 -sec delay training, with the exception that a 1 -sec delay followed the completion of the response requirement to the upper picture. The subjects were trained until performance was $\geq 80 \%$ in a single session. Immediately upon completing this phase of training, transfer testing began in the next session.

Novel item transfer testing. Each transfer session consisted of 90 baseline training ( 45 same, 45 different) trials plus 5 same and 5 different transfer trials. Novel stimuli, never before seen, were presented on transfer trials. The 10 transfer trials were pseudorandomly placed within each session following the 7th trial. Performance on transfer trials was reinforced like that on baseline trials. Transfer testing was conducted for six consecutive sessions.

\section{Results and Discussion}

\section{Acquisition}

Overall performance for the 0 -sec delay $(M=76.41 \%$, $S E M=1.5)$ and $1-\sec$ delay $(M=77.62 \%, S E M=4.7)$ training was good. With 0 -sec delay training, 3 pigeons reached the $80 \%$ criterion in their first session. One pigeon (K1812) was disrupted (56\%) by the introduction of the delay, but rebounded in the following session (75\%) and reached the $80 \%$ performance criterion in six sessions. With 1-sec delay training, all of the pigeons reached the performance criterion by seven sessions.

The introduction of these delays provides an opportunity to compare initial acquisition during these delay conditions to terminal performance of the simultaneous task. Transfer from the simultaneous to the sequential tasks would provide evidence against the translationalsymmetry hypothesis. Figure 2 shows the mean percentage correct for the last simultaneous, first 0 -sec delay, last 0 -sec delay, and first 1 -sec delay training sessions. As is shown, all of the subjects transferred from the simultaneous $\mathrm{S} / \mathrm{D}$ procedure to the 0 -sec delay condition. Mean performance on the last session of the simultaneous S/D condition $(M=81 \%, S E M=2.7)$ was statistically equivalent to mean performance on the first session of 0-sec sequential S/D condition $(M=76.3 \%, S E M=6.8)$ [paired $t$ test, $t(3)=1.04, p>.37$ ]. All of the subjects transferred well from the 0 -sec delay condition to the 1-sec delay condition. Mean performance on the last session of the 0 -sec sequential S/D condition $(M=83 \%, S E M=1.15)$ was statistically higher than mean performance on the first session of the $1-\sec$ sequential S/D condition $(M=73.50 \%$,

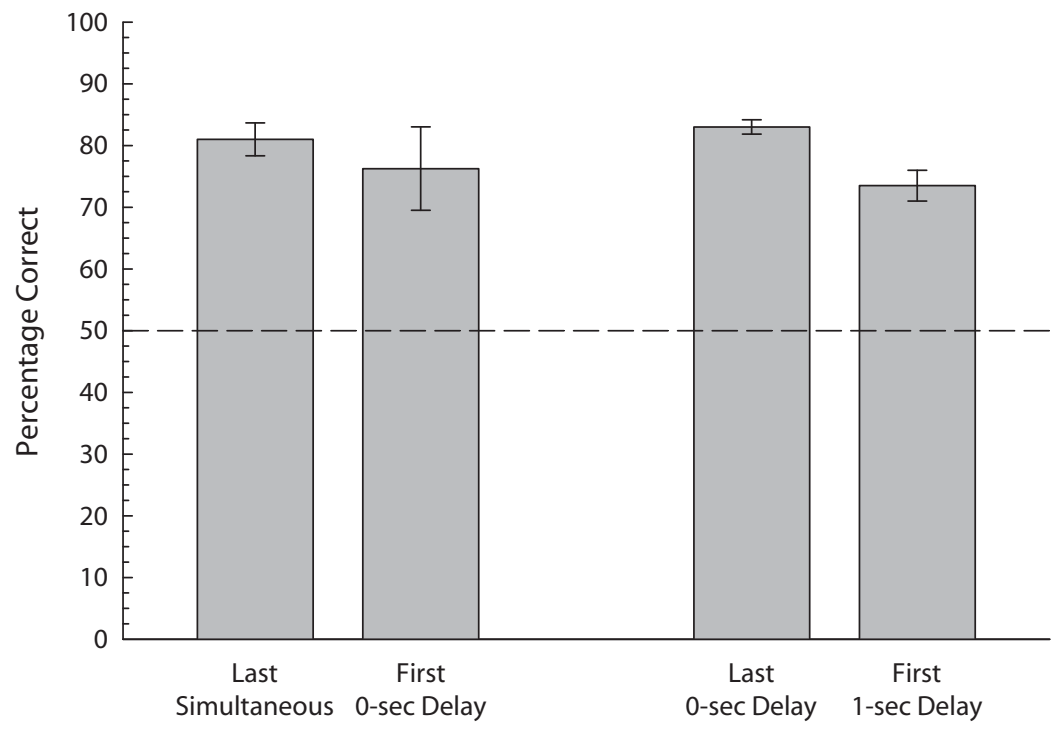

Figure 2. Mean performance for the 4 pigeons during the last session of simultaneous, first session of 0-sec delay, last session of 0-sec delay, and first session of 1-sec delay same/different training. The dashed line represents chance performance, and error bars represent standard errors of the mean. 




Figure 3. Mean performance on same and different trials for the 4 pigeons during the last session of simultaneous, first session of 0-sec delay, last session of 0-sec delay, and first session of 1-sec delay same/different training. The dashed line represents chance performance, and error bars represent standard errors of the mean.

$S E M=2.5)$ [paired $t$ test, $t(3)=3.3, p<.05$ ] but was statistically greater than chance (50\%) [one-sample $t$ test, $t(3)=9.4, p<.004]$. This last result indicates that although there was a slight disruption in transitioning to the 1 -sec delay condition, the pigeons performed well above chance level (50\% correct).

To analyze for a default strategy, we examined percentage correct for same and different trials for the last simultaneous, first 0 -sec delay, last 0 -sec delay, and first 1 -sec delay sessions for each of the subjects, as is illustrated in Figure 3. A two-way repeated measures ANOVA of condition (last simultaneous, first 0 -sec delay, last 0 -sec delay, first 1 -sec delay sessions) $\times$ trial type (same, different) on percentage correct yielded no main effect of condition $[F(3,9)=$ $1.14, p=.38]$ or trial type $[F(1,3)=3.16, p=.17]$ and no interaction $[F(3,9)<1, p=.73]$. No statistical difference for same and different trials suggests the absence of a default strategy and contradicts the translational-symmetry hypothesis. In fact, the trend was somewhat in the opposite direction of the predicted outcome by the translationalsymmetry hypothesis (different, $M=71.75 \%, S E M=3.7$; same, $M=85.12 \%$, SEM $=1.8$ ).

The above analyses provide evidence against the translational-symmetry account of abstract-concept learning; however, it might be argued that the pigeons rapidly learned a new strategy. To rule out relearning when the stimuli were presented sequentially, we compared the total number of trials for each pigeon to reach the performance criteria with the 0 -sec and 1-sec delay (sequential) with the total number of trials required for these same pigeons to reach the performance criteria through set-size expansion to the 1,024 set size (simultaneous). The simultaneous condition includes all of the training trials for each set-size acquisition (i.e., 8, 16, 32, 64, 128, 256, 512, and $1,024)$. There were great savings in acquisition when the pigeons transitioned to the sequential conditions $(M=800$ trials, $S E M=187.1$ ) relative to their initial learning of the simultaneous $\mathrm{S} / \mathrm{D}$ task $(M=6,950$ trials, $S E M=635.7)$ [paired $t$ test, $t(3)=13.9, p<.001]$ ] This comparison of simultaneous and sequential acquisition performance suggests that the pigeons were not learning the sequential task via a new strategy (cf. Cook \& Wasserman, 2007). This conclusion is further supported by the pigeons' ability to transfer to novel stimuli under the delayed conditions, as is shown in the next section.

\section{Novel Item Transfer}

Baseline and transfer performance during the six sessions of novel item transfer testing with a 1-sec delay for each pigeon are shown in Figure 4. Transfer performance was equivalent to baseline performance, as was confirmed by a repeated measures ANOVA on mean percentage correct for each session with trial type (baseline, transfer) as a factor that revealed no main effect $[F(1,23)=1.68$, $p>$.2]. Also, there was no change in transfer performance across the test sessions $[F(5,15)=0.37, p>.8]$ and no difference between performance on same and different transfer trials $[F(1,23)=2.37, p>.14]$. The pigeons' transfer was statistically equivalent to their initial transfer with the 1,024 items in the simultaneous procedure, as was confirmed by a repeated measures ANOVA for each session $[F(1,23)=3.15, p>.09]$. These results offer further evidence that the discrimination processes used by the pigeons when they transferred from the simultaneous to the sequential presentation of the stimuli were unchanged.

\section{EXPERIMENT 2}

Experiment 1 showed that transfer from a simultaneous two-item S/D task to a 0 - and a 1-sec delay S/D task did not 




Figure 4. Mean performance for baseline and transfer averaged over the six transfer sessions for each pigeon during 1 -sec delay transfer. The dashed line represents chance performance, and error bars represent standard errors of the mean.

disrupt performance, and there was no evidence that these subjects defaulted to different choices, contrary to what would be expected by the translational-symmetry hypothesis. In addition, the pigeons showed a high level (76\%) of transfer to novel items that was not statistically different from baseline under these delayed conditions. These results show a continuity of processing by these pigeons from the simultaneous to the delayed tasks and suggest that translational symmetry or other emergent features were not controlling performance in our two-item S/D task.

Although the results from Experiment 1 were encouraging, it is difficult to be absolutely sure that $1 \mathrm{sec}$ is sufficient to render translational symmetry imperceptible for pigeons. Therefore, in Experiment 2, we increased the delay further, initially to $2 \mathrm{sec}$ and then to $6 \mathrm{sec}$. Introducing the longer delays one at a time allowed us to study how the subjects would distribute their responses when new, longer delays were introduced. If the subjects were defaulting to different responses in the absence of a controlling translationalsymmetry cue, a different bias should appear as the delay became long enough that the translational-symmetry cue was no longer apparent. Hence, the translational-symmetry hypothesis predicts increases in accuracy on different trials but decreases in accuracy on same trials - in other words, asymmetrical retention functions (e.g., Singer, Klein, \& Zentall, 2006; Wixted \& Gaitan, 2004).

\section{Method}

\section{Subjects, Apparatus, and Stimuli}

Three of the pigeons from Experiment 1 were used in Experiment 2. The 4th pigeon fell ill and could not participate in Experiment 2. The apparatus and stimuli were those from Experiment 1.

\section{Variable-Delay Testing}

Variable-delay testing immediately followed the novel item transfer in Experiment 1. The delay values trained and tested were sys- tematically increased. First, delays of 0,1 , and 2 -sec were tested within a session. Each session consisted of 90 trials (45 same, with 15 trials for each of the three delays; 45 different, with 15 trials for each of the three delays). Testing was conducted for 2 sessions. Next, delays of $0,1,2$, and $6 \mathrm{sec}$ were tested within a session. Each session consisted of 96 trials (48 same, with 12 trials for each of the four delays; 48 different, with 12 trials for each of the delays). Testing was conducted for 12 sessions. Other details were identical to Experiment 1.

\section{Results and Discussion}

Figure 5 shows the overall percentage correct (triangles) for the initial and expanded variable-delay tests for same and different performance. Neither variable-delay test showed a precipitous decrement in performance. Instead, performance was well above chance $(50 \%)$. These results were confirmed by separate one-sample $t$ tests comparing each overall percentage correct at each delay in Figure 5 with chance [all $t \mathrm{~s}(2)>5.3, p \mathrm{~s}<.034$ ].

There were no differences on same and different trials across delays for $0-, 1-$, and 2 -sec variable-delay testing (left panel of Figure 5). There were no trends in the functions across sessions, although the overall accuracy increased slightly $(1.8 \%)$ between the first and last day of testing. Most important, there were no changes in same and different performance across the 0-, 1-, and 2-sec delays (left panel of Figure 5). No statistical differences between same and different performance across delays indicates that the subjects did not default to different responses at longer delays, as would be predicted by the translationalsymmetry hypothesis. These findings were confirmed by a three-way repeated measures ANOVA of delay $(0,1$, $2 \mathrm{sec}) \times$ trial type $($ same, different $) \times \operatorname{session}(1,2)$, which yielded only a main effect of session $[F(1,2)=21.6, p<$ .05 ; all other $F \mathrm{~s}<3.1, p \mathrm{~s}>.16]$. The $0-, 1-, 2-$, and 6-sec variable-delay test (right panel of Figure 5) did show a drop 




Figure 5. Overall mean performance (triangles) and same and different performance (circles) during testing with 0-, 1-, and 2-sec delays (left panel) and 0-, 1-, 2-, and 6-sec delays (right panel). The dashed line represents chance performance, and error bars represent standard errors of the mean.

in performance at the 6-sec delay (relative to the $0-, 1-$, and 2 -sec delays), but there was no significant difference between same and different trials. Most important, there was no evidence for asymmetrical retention functions, and no significant change across sessions. These findings further indicate that subjects did not increase different responding and decrease same responding with an increase in delay, as would be predicted by the translational-symmetry hypothesis. There was an increase (9\%) in percentage correct between the first and last day of testing, but again, there was no change in the functions. These results were confirmed by a three-way repeated measures ANOVA of delay $(0,1$, $2,6) \times$ trial type (same, different) $\times$ session $(1-12)$, which yielded only a main effect of session $[F(1,11)=5.2, p<$ $.01]$ and delay $[F(3,6)=9.7, p<.01 ;$ all other $F \mathrm{~s}<1.25$, $p \mathrm{~s}>.22]$.

\section{GENERAL DISCUSSION}

The experiments reported in this article provide converging evidence that translational symmetry does not account for the pigeons' abstract-concept learning in our two-item S/D task. The pigeons showed excellent transitions from a simultaneous to sequential presentation of two items. They performed smooth transitions from simultaneous to 0 -sec and from 0 - to 1 -sec delays (Experiment 1) and from 1- to 2-sec and from 2- to 6-sec delays (Experiment 2). In addition, their accurate novel-item transfer at a 1 -sec delay demonstrated that translational symmetry was unlikely to be the controlling cue in transfer and abstractconcept learning. These conclusions are further bolstered by the lack of any tendency to respond different as the delay was increased. If translational symmetry had been the controlling cue for same responses, the strength of this cue should have dissipated with delay increases, and the tendency to respond different (a different bias) should have increased with delay increases.

If the pigeons in our experiment had learned to discriminate training stimulus pairs via translational symmetry, such a strategy should have worked equally well with novel items (cf. Cook et al., 1995; Wasserman et al., 1995). When the pigeons learned the initial task with a small set of 8 items, they showed no transfer to novel items. This result in itself is evidence against learning translational symmetry, because if they had learned translational symmetry, they should have shown considerable novel-stimulus transfer. Indeed, as the training set was expanded to $32,64,128,256,512$, and 1,024 items, these subjects gradually showed better transfer. This gradual increase in transfer is important, because if at any point they had switched to relying on translational symmetry, transfer performance should have jumped to a high level relative to baseline performance.

We have trained 20 rhesus monkeys and 4 capuchin monkeys in two-item S/D tasks virtually identical to the one shown here for pigeons. In all cases, transition from a simultaneous to a sequential S/D procedure was seamless for these subjects (Wright \& Katz, 2007; Wright, Rivera, Katz, \& Bachevalier, 2003). Capuchin and rhesus monkeys, like the pigeons, also showed excellent transfer to novel stimuli in the delayed S/D task. Thus, we conclude that all of these species learned this particular two-item S/D task in the same way. They compared the two items presented (either simultaneously or sequentially) and made a choice on the basis of the relationship between these two items. It is learning the relationship of same or different that forms the basis of higher order abstractconcept learning, and this relational learning ability appears to be shared across species as diverse as primates and avians. 


\section{AUTHOR NOTE}

This research was supported by NIH Grants MH-061798 and MH072616 and by NSF Grant IBN-0316113. We wish to thank Kent Bodily, Michelle Hernández, and Jacquelyne Rivera for their careful assistance in the research. Correspondence concerning this article should be addressed to J. S. Katz, Department of Psychology, 226 Thach Hall, Auburn University, Auburn, AL 36849; B. R. Sturz, Department of Psychology, Armstrong Atlantic State University, 229 Science Center, Savannah, GA 31419; or A. A. Wright, Department of Neurobiology and Anatomy, University of Texas Medical School at Houston, P.O. Box 20708, Houston, Texas 77225 (e-mail: katzjef@auburn.edu, bradley.sturz@armstrong.edu, or anthony.a.wright@uth.tmc.edu).

\section{REFERENCES}

Blaisdell, A. P., \& CooK, R. G. (2005). Two-item same-different concept learning in pigeons. Learning \& Behavior, 33, 67-77.

Cook, R. G., Cavoto, K. K., \& Cavoto, B. R. (1995). Same/different texture discrimination and concept learning in pigeons. Journal of Experimental Psychology: Animal Behavior Processes, 21, 253-260.

Cook, R. G., Katz, J. S., \& Cavoto, B. R. (1997). Pigeon samedifferent concept learning with multiple stimulus classes. Journal of Experimental Psychology: Animal Behavior Processes, 23, 417-433. doi:10.1037/0097-7403.23.4.417

CooK, R. G., Kelly, D. M., \& KatZ, J. S. (2003). Successive two-item same-different discrimination and concept learning by pigeons. Behavioral Processes, 62, 125-144. doi:10.1016/S0376-6357(03)00022-6

CoOK, R. G., \& WASSERMAN, E. A. (2006). Relational discrimination learning in pigeons. In E. A. Wasserman \& T. R. Zentall (Eds.), Comparative cognition: Experimental explorations of animal intelligence (pp. 207-324). Oxford: Oxford University Press.

Cook, R. G., \& Wasserman, E. A. (2007). Learning and transfer of relational matching-to-sample by pigeons. Psychonomic Bulletin \& Review, 14, 1107-1114.

Elmore, L. C., Wright, A. A., Rivera, J. J., \& Katz, J. S. (2009). Individual differences: Either relational learning or item-specific learning in a same/different task. Learning \& Behavior, 37, 204-213. doi:10.3758/LB.37.2.204

Humphreys, G. W., Quinlan, P. T., \& RidDOch, M. J. (1989). Grouping processes in visual search: Effects with single- and combined-feature targets. Journal of Experimental Psychology: General, 118, 258-279.

JAMES, W. (1950). The principles of psychology (Vol. 1). New York: Dover. (Original work published 1890)

Katz, J. S., \& Wright, A. A. (2006). Same/different abstract-concept learning by pigeons. Journal of Experimental Psychology: Animal Behavior Processes, 32, 80-86. doi:10.1037/0097-7403.32.1.80

KatZ, J. S., Wright, A. A., \& Bodily, K. D. (2007). Issues in the comparative cognition of abstract-concept learning. Comparative Cognition \& Behavior Reviews, 2, 79-92.

MACH, Е. (1980). Contributions to the analysis of the sensations (C. M. Williams, Trans). La Salle, IL: Open Court. (Original work published 1890)

Mackintosh, N. J. (2000). Abstraction and discrimination. In C. Heyes \& L. Huber (Eds.), The evolution of cognition (pp. 123-141). Cambridge, MA: MIT Press.
Nakamura, T., Wright, A. A., Katz, J. S., Bodily, K. D., \& Sturz, B. R. (2009). Abstract-concept learning carryover effects from the initial training set in pigeons (Columba livia). Journal of Comparative Psychology, 123, 79-89. doi:10.1037/a0013126

Penn, D. C., Holyoak, K. J., \& Povinelli, D. J. (2008). Darwin's mistake: Explaining the discontinuity between human and nonhuman minds. Behavioral \& Brain Sciences, 31, 109-130.

Piaget, J., \& Inhelder, B. (1969). The psychology of the child (H. Weaver, Trans.). New York: Basic Books. (Original work published 1966)

Premack, D. (1978). On the abstractness of human concepts: Why it would be difficult to talk to a pigeon. In S. H. Hulse, H. Fowler, \& W. K. Honig (Eds.), Cognitive processes in animal behavior (pp. 423451). Hillsdale, NJ: Erlbaum.

Santiago, H. C., \& Wright, A. A. (1984). Pigeon memory: Same/ different concept learning, serial probe recognition acquisition, and probe delay effects on the serial-position function. Journal of Experimental Psychology: Animal Behavior Processes, 10, 498-512. doi:10.1037/0097-7403.10.4.498

Singer, R. A., Klein, E. D., \& Zentall, T. R. (2006). Use of a singlecode/default strategy by pigeons to acquire duration sample discriminations. Learning \& Behavior, 34, 340-347.

TYLeR, C. W. (2002). Human symmetry perception and its computational analysis. Mahwah, NJ: Erlbaum.

Wasserman, E. A., Hugart, J. A., \& KirkPatrick-Steger, K. (1995). Pigeons show same-different conceptualization after training with complex visual stimuli. Journal of Experimental Psychology: Animal Behavior Processes, 21, 248-252.

WiXTED, J. T., \& GaITAN, S. C. (2004). Stimulus salience and asymmetric forgetting in the pigeon. Learning \& Behavior, 32, 173-182.

Wright, A. A., \& KATZ, J. S. (2006). Mechanisms of same/different concept learning in primates and avians. Behavioural Processes, $\mathbf{7 2}$ 234-254. doi:10.1016/j.beproc.2006.03.009

Wright, A. A., \& KATZ, J. S. (2007). Generalization hypothesis of abstract-concept learning: Learning strategies and related issues in Macaca mulatta, Cebus apella, and Columba livia. Journal of Comparative Psychology, 121, 387-397. doi:10.1037/0735-7036.121.4.387

Wright, A. A., \& KATZ, J. S. (2009). A case for restricted-domain relational learning. Psychonomic Bulletin \& Review, 16, 907-913. doi: 10.3758/PBR.16.5.907

Wright, A. A., Rivera, J. J., Katz, J. S., \& Bachevalier, J. (2003). Abstract-concept learning and list-memory processing by capuchin and rhesus monkeys. Journal of Experimental Psychology: Animal Behavior Processes, 29, 184-198. doi:10.1037/0097-7403.29.3.184

YounG, M. E., \& Wasserman, E. A. (1997). Entropy detection by pigeons: Response to mixed visual displays after same-different discrimination training. Journal of Experimental Psychology: Animal Behavior Processes, 23, 157-170.

Young, M. E., \& Wasserman, E. A. (2001). Evidence for a conceptual account of same-different discrimination learning in the pigeon. Psychonomic Bulletin \& Review, 8, 677-684.

(Manuscript received for publication July 7, 2009; accepted for publication July 9, 2009.) 